\title{
Author Correction: An analytical framework for interpretable and generalizable single-cell data analysis
}

Jian Zhou (1) and Olga G. Troyanskaya (1)

Correction to: Nature Methods https://doi.org/10.1038/s41592-021-01286-1, published online 1 November 2021.

In the version of this article originally published, there was an error in a formula in the fourth sentence of the third paragraph of Methods. In the text now reading "The analytical solution to the optimization problem is $Z=(I+\lambda L)^{-1} X W$, where $W$ represents the top $d$ eigenvectors of $X^{\mathrm{T}}(I+\lambda L)^{-1} X$, where $d$ is the dimensionality of $Z$," an extraneous equals sign appeared between " $X^{\mathrm{T}}$ ” and " $(I+\lambda L)^{-1} X$." The error has been corrected in the HTML and PDF versions of the article.

Published online: 14 February 2022

https://doi.org/10.1038/s41592-022-01421-6

(c) The Author(s), under exclusive licence to Springer Nature America, Inc. 2022 